\title{
Comparison of Posterior Lumbar Interbody Fusion and Posterolateral Lumbar Fusion in Monosegmental Vacuum Phenomenon within an Intervertebral Disc
}

\author{
Vibhu Krishnan Viswanathan ${ }^{1}$, Surabhi Subramanian ${ }^{2}$ \\ ${ }^{I}$ Department of Orthopedics, Spine Centre, Indian Spinal Injuries Centre, New Delhi, India \\ ${ }^{2}$ Department of Radiology, Government Medical College, Nagpur, India
}

We read with great interest the article by An et al. [1] titled "Comparison of Posterior Lumbar Interbody Fusion and Posterolateral Lumbar Fusion in Monosegmental Vacuum Phenomenon within an Intervertebral Disc". The authors analyzed retrospectively collected data involving 84 patients and described their experience with posterolateral and posterior lumbar inter-vertebral fusion in situations of mono-segmental inter-vertebral disc vacuum. This issue remains controversial and the authors' description is definitely an eye-opener to the readers. It is a wellwritten article and has fore-grounded some of the major concerns of the contemporary medical fraternity. We do have some queries to the authors regarding this study:

(1) The authors have compared the results of two different fusion modalities in patients with X-ray evidence of vacuum phenomenon. However, there is no mention of magnetic resonance imaging (MRI) findings in these patients including extruded or protruded disc fragments, degree of disc degeneration [2], foraminal stenosis, facetal hypertrophy and site of nerve root compression. We believe that these factors also play a major role in treatment decision-making, as inter-body fusions may be considered superior in situations where disc removal, facetectomy or foraminal height restoration and decompression are necessary. (2) Did the authors obtain dynamic radiographs or whole spine films as part of their surgical planning? Stress views may indicate subtle instabilities that can modify management strategies. The need for limited computed tomography (CT) scans to rule out subtle lysis at the involved levels may also be considered in certain patients. (3) Although patients with spondylolisthesis and scoliosis were excluded, the X-rays included in the article show cases with grade 1 spondylolisthesis (patient who underwent inter-body fusion) and degenerative scoliosis (postero-lateral fusion case). (4) Liao et al. [3] indicated that interbody fusion is associated with better restoration of radiological parameters including segmental lordosis, translational alignment and greater regaining of disc height. Did the authors consider any further criteria like decreased disc height or sagittal mal-alignment to subgroup the patients with vacuum disc phenomenon, in which the two fusion procedures can result in different outcomes? (5) As mentioned by the authors, details regarding the bone mineral density can alter the management protocol in

\footnotetext{
Received Jun 6, 2016; Accepted Jun 8, 2016

Corresponding author: Vibhu Krishnan Viswanathan

Department of Orthopedics, Spine Centre, Indian Spinal Injuries Centre,

Sector C, Vasant Kunj, New Delhi - 110070, India

Tel: +91-99-4052-8382, E-mail: drvibu007@gmail.com
} 
these patients. It is unfortunate that this information was not available. (6) There is a minor error in page 97, where the operative time and blood loss have been mentioned to be greater in the posterolateral fusion group. However, elsewhere in the article, it has been mentioned otherwise. We believe this discrepancy is entirely typographical.

The current article has discussed one of the most relevant issues in fusion procedures for degenerative spine disorders. The author's contribution is a laudable effort. We would really appreciate their comments on our queries.

\section{Conflict of Interest}

No potential conflict of interest relevant to this article was reported.

\section{References}

1. An KC, Kong GM, Park DH, Baik JM, Youn JH, Lee WS. Comparison of posterior lumbar interbody fusion and posterolateral lumbar fusion in monosegmental vacuum phenomenon within an intervertebral disc. Asian Spine J 2016;10:93-8.

2. Taher F, Essig D, Lebl DR, et al. Lumbar degenerative disc disease: current and future concepts of diagnosis and management. Adv Orthop 2012;2012:970752.

3. Liao JC, Lu ML, Niu CC, Chen WJ, Chen LH. Surgical outcomes of degenerative lumbar spondylolisthesis with anterior vacuum disc: can the intervertebral cage overcome intradiscal vacuum phenomenon and enhance posterolateral fusion? J Orthop Sci 2014;19: 851-9. 